\title{
Prominent entrapment of respiratory epithelium in primary and metastatic intrapulmonary non-epithelial neoplasms: a frequent morphological pattern closely mimicking adenofibroma and other biphasic pulmonary lesions
}

\author{
Ramona Erber ${ }^{1} \cdot$ Florian Haller $^{1} \cdot$ Arndt Hartmann $^{1} \cdot$ Abbas Agaimy $^{1}$ \\ Received: 11 October 2019 / Revised: 10 February 2020 / Accepted: 8 March 2020 / Published online: 19 March 2020 \\ (C) The Author(s) 2020
}

\begin{abstract}
As one of the most common target organs for hematogenous spread from diverse cancers, biopsy interpretation of lung tumors is complicated by the challenging question of primary versus metastatic and by frequent entrapment of native respiratory glands. Nevertheless, the literature dealing with this issue is surprisingly sparse and no single study has been devoted to this topic. We reviewed 47 surgical lung specimens of non-epithelial neoplasms (38 metastases, mainly from sarcomas and 9 primary lesions) for frequency and pattern of intralesional epithelial entrapment. Respiratory epithelium entrapment was noted in 23/47 (49\%) cases (diffuse in 15 and peripheral in 8). Entrapped glands frequently showed prominent regenerative and reactive changes mimicking neoplastic glands. Based on cellularity of the mesenchymal component and the extent, distribution and shape of entrapped respiratory glands, four morphological patterns were recognized: paucicellular sclerosing low-grade neoplasms containing leaflet-like glands indistinguishable from adenofibroma and fibroepithelial hamartomas $(n=11)$, and biphasic cellular lesions mimicking adenomyoepithelioma $(n=1)$, biphasic synovial sarcoma $(n=2)$, and pleuropulmonary blastoma $(n=1)$. Only a single genuine pulmonary adenofibroma was identified. This study highlights frequent respiratory epithelium entrapment in diverse non-epithelial lung tumors, both primary and metastatic. Recognition of this finding and use of adjunct IHC combined with clinical history should help to avoid misinterpretation as primary pulmonary biphasic neoplasm or as harmless adenofibroma. The vast majority of morphologically defined lung adenofibromas represent adenofibroma-like variants of histogenetically diverse entities so that a diagnosis of adenofibroma should be rendered only very restrictively and then as a diagnosis by exclusion.
\end{abstract}

Keywords Sarcoma $\cdot$ Lung metastasis $\cdot$ Adenofibroma $\cdot$ Fibroadenoma $\cdot$ Lung $\cdot$ Nephroblastoma $\cdot$ Osteosarcoma $\cdot$ Germ cell tumor $\cdot$ Adenomyoepithelioma $\cdot$ Hamartoma

\section{Introduction}

The lungs represent major target organs for metastatic deposits from malignant neoplasms of diverse histogenetic origin from different anatomic sites. Sarcomas in particular are known to have significant predilection for hematogenous spread to the lungs. Accordingly, thoracic imaging represents an integral

Abbas Agaimy

abbas.agaimy@uk-erlangen.de

1 Institute of Pathology, Friedrich-Alexander University Erlangen-Nürnberg (FAU), University Hospital, Krankenhausstrasse 8-10, 91054 Erlangen, Germany part of routine staging investigations for newly diagnosed malignant neoplasms and on follow-up for patients with a history of malignancy. As a consequence of increasing use of highresolution imaging and increasing frequency of thoracoscopic surgery, incidental pulmonary nodules are being increasingly discovered and excised. Familiarity with the diverse patterns of primary and metastatic intrapulmonary non-epithelial neoplasms is mandatory for distinguishing benign from malignant and primary from metastatic diseases.

A subset of incidentally discovered pulmonary nodules, whether solitary or multiple, represent hamartomatous lesions or benign mesenchymal neoplasms, chondroid hamartomas being the most frequent. Pulmonary adenofibroma (PAF) is an exceptionally rare benign fibroepithelial lesion that closely 
recapitulates Müllerian adenofibroma of the genital tract [1] or fibroadenoma of the breast [2]. Since its first description by Scarff and Gowar in 1944 [3] and delineation as distinctive entity by Suster and Moran in 1993 [1], only a few cases of genuine PAFs have been described $[4,5]$.

Although entrapment of native pulmonary epithelium by metastatic neoplasms in the lung is well known, in our experience this phenomenon and the pitfalls related to it have not received sufficient attention in the surgical pathology literature, possibly explaining its under-recognition among general surgical pathologists in routine practice. Encountering several cases that have illustrated the difficulty and confusion related to this finding, we decided to review our files for nonepithelial pulmonary lesions, both primary and metastatic, to critically address and illustrate this histological finding and discuss the sources of pitfall in the context of the differential diagnoses of biphasic pulmonary lesions.

\section{Material and methods}

We searched our routine and consultation files for surgically excised lung metastases from malignant neoplasms other than carcinomas (including sarcoma of different types, embryonal tumors, and germ cell tumors) diagnosed in our institution between 2012 and 2018. Furthermore, our archive was searched for lung lesions diagnosed as adenofibroma, perivascular epithelioid cell tumor (PEComa), and solitary fibrous tumors (SFT). Diagnosis, site of origin, patient's age, gender, and history of malignancy were drawn from the original pathology records. To have a representative overview of this morphological phenomenon, only surgical specimens were analyzed. In each case, all resected lung metastases have been reviewed and the presence, pattern, and extent of entrapped lung epithelium have been recorded (described as diffuse resulting in a biphasic pattern throughout versus focal and peripheral). Those cases with diffuse entrapment of respiratory epithelium were then used for further analysis. In most cases, immunohistochemical staining (CK7, TTF1, and NapsinA, in addition to other markers based on the exact diagnosis in a given case) was available. The overall histological appearance of the lesion was then described as adenofibroma-like, adenomyoepithelioma-like, biphasic synovial sarcoma-like, and other non-descript biphasic patterns.

\section{Results}

\section{General clinical and demographic features}

Forty-seven patients were retrieved for histological analysis. These comprised 38 patients with pulmonary metastases $(81 \%)$ and 8 cases of primary pulmonary non-epithelial lesions (6 primary intrapulmonary SFTs, one SMARCB1deficient fibromyxoid lung neoplasm of uncertain histogenesis, and one pulmonary adenofibroma). One PEComa could not be determined whether it is primary or metastatic due to lack of detailed clinical information. The 38 cases of lung metastases were from different sarcoma types $(n=35)$, germ cell tumors $(n=2)$, and Wilms tumor $(n=1)$. Age of the patients ranged between 2 and 84 years (median 52 years, mean 47 years). Twenty-one patients were females (44.7\%). Eighteen patients suffered from $\geq 2$ lung metastases; one patient had $>30$ small lung metastases that were resected. Patient characteristics are shown in Table 1.

\section{Frequency of respiratory/alveolar epithelium entrapment}

In total, 23 out of 47 cases (49\%) showed entrapment of respiratory/alveolar epithelium with variable extent. In eight of these cases, entrapped pulmonary epithelium was seen mainly in the peripheral portion of the tumor and was clearly evident as secondary entrapment of lung tissue as the tumor grows peripherally. The remainder $(n=15)$, which represent the basis of the subsequent morphological analysis, showed prominent entrapped alveolar and respiratory epithelium that was seen throughout the lesion albeit to varying extent. These entrapped glands frequently showed reactive/regenerative appearance with occasional hobnail-like nuclear prominence, vesicular chromatin, and irregular configuration mimicking neoplastic glands, but lacked significant cytological atypia. The gland size varied greatly from small acinar-type glands or microcystic spaces lined by flattened epithelial cells and containing mucoid secretion to branching leaflet-like papillary spaces. The overall appearance of these glands was different from normal alveoli, thus enhancing their confusion with true neoplastic glands. Based on the cellularity of the background neoplasm, four different histological patterns were recognized: (1) lesions closely mimicking PAF (11/15); (2) those closely mimicking adenomyoepithelioma/epithelialmyoepithelial carcinoma (1/15); (3) biphasic synovial sarcoma-like (2/15); and (4) pulmonary blastoma-like (1/15) patterns (Figs. 1, 2, and 3, Table 2).

\section{Adenofibroma-like pattern}

This pattern (seen in 11/15 cases; $73 \%$ ) was characterized by the presence of a diffuse component of branching leaflet-like variably dilated glands throughout the lesion imparting a characteristic adenofibroma-like, phylloides-like or fibroepithelial hamartoma-like pattern. This feature (which is restricted to spindle cell neoplasms with low-grade histological features and paucicellular sclerosing stroma) was seen in 4 primary intrapulmonary lesions (2 SFTs, 1 unclassified myxoid lesion, and 1 pulmonary adenofibroma), 1 PEComa (not clear if 


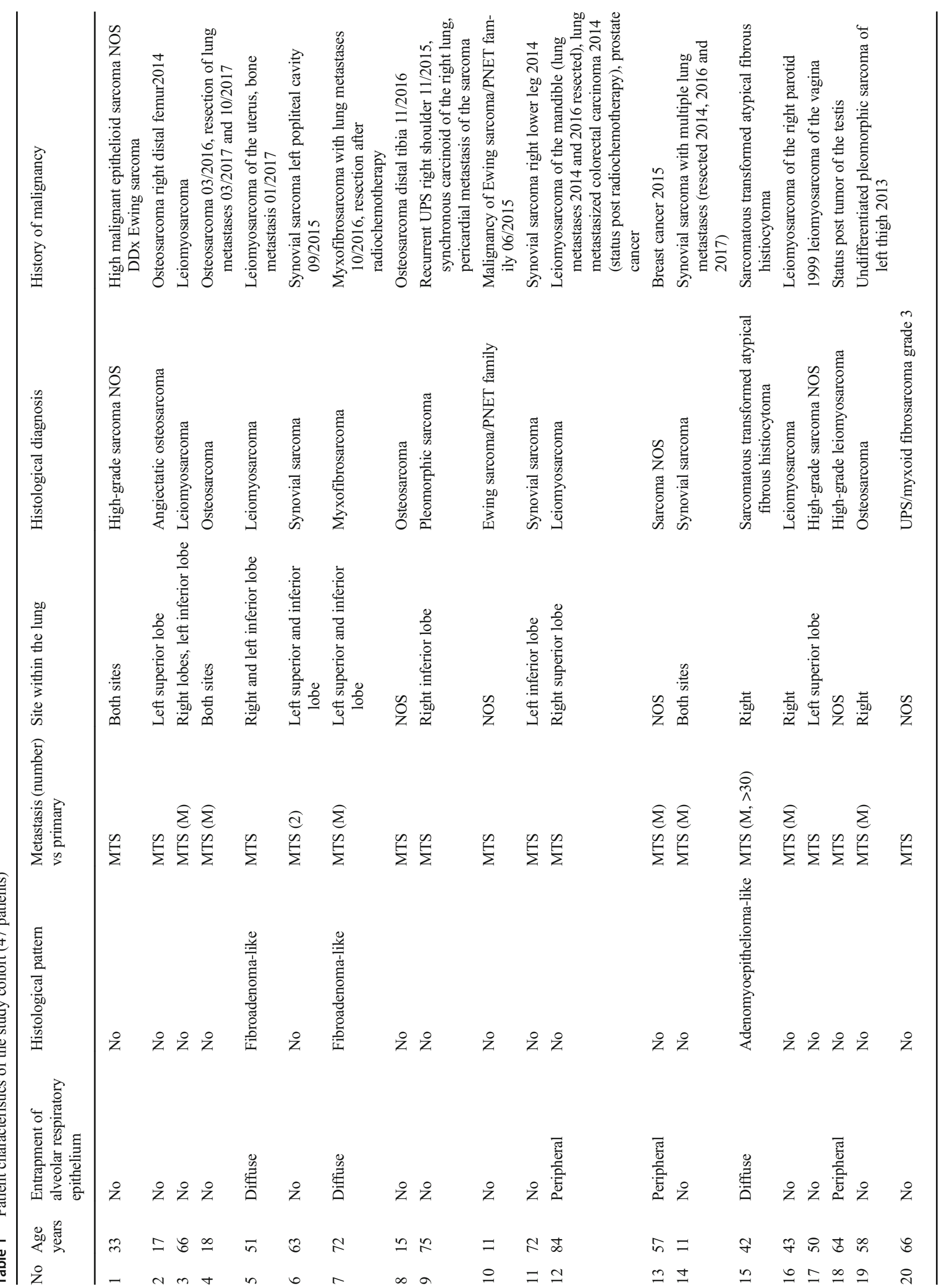




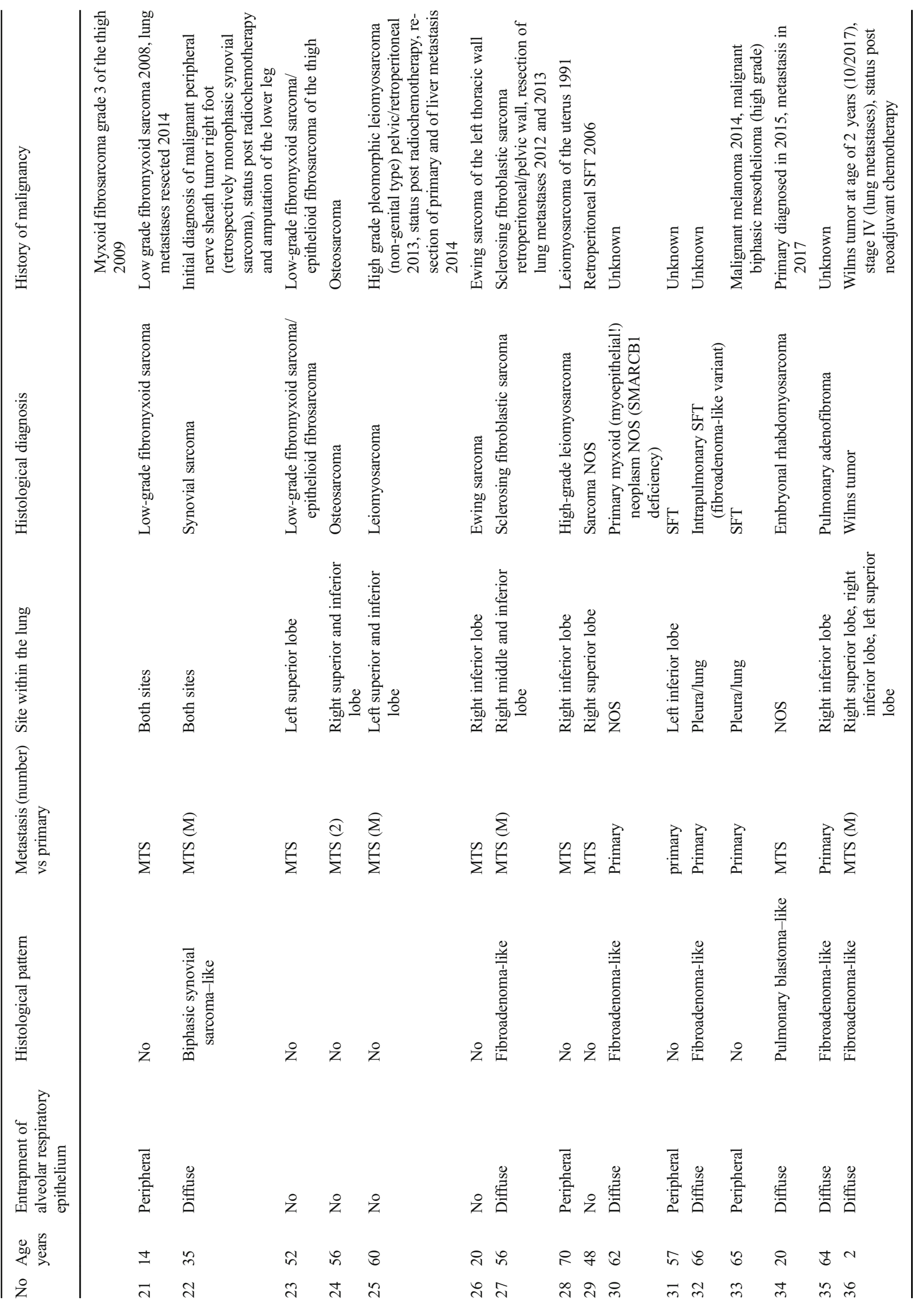




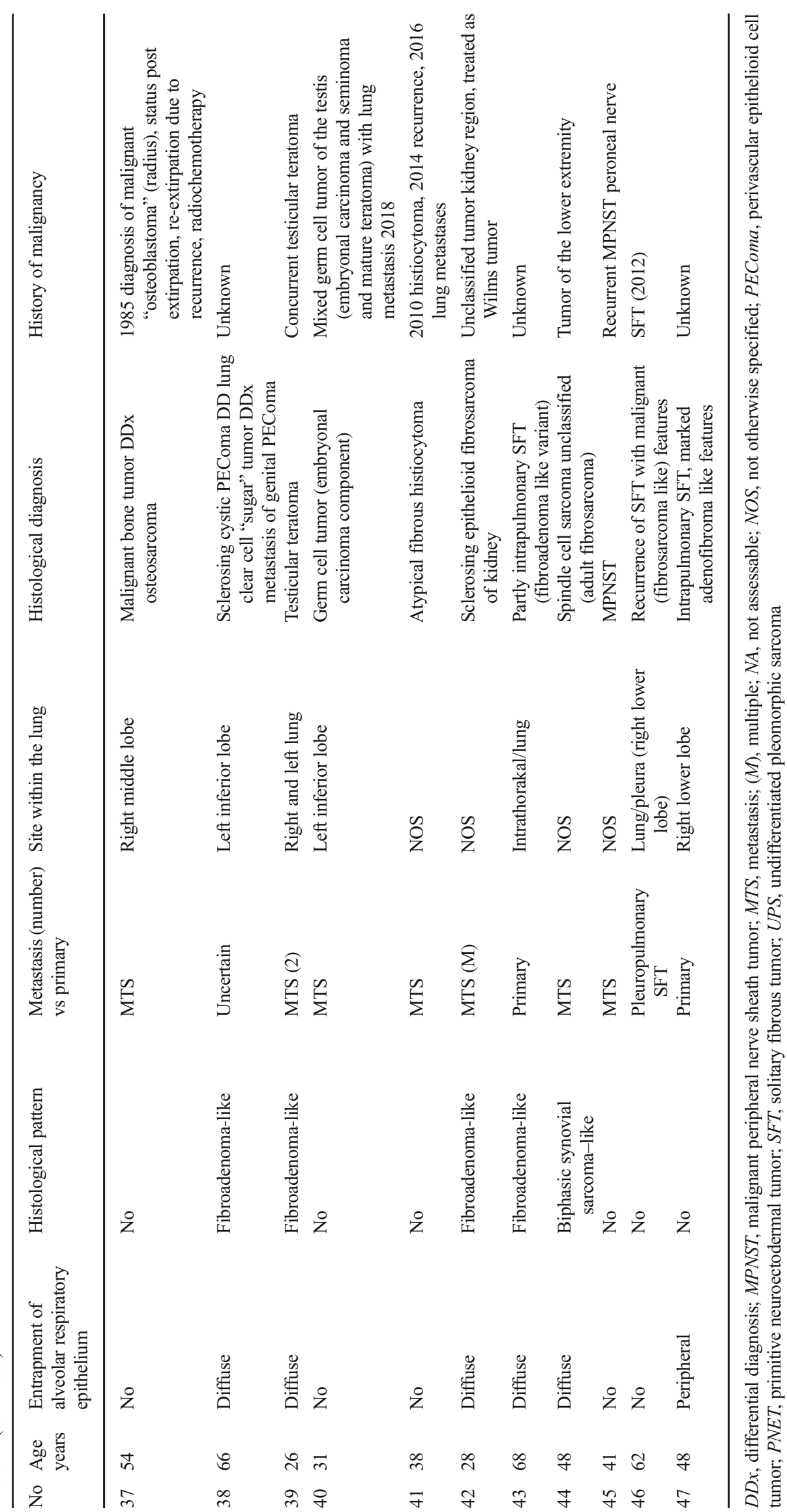


Fig. 1 a The single genuine case of pulmonary adenofibroma presented as well circumscribed whitish nodule (lower field). Histology showed biphasic fibroadenoma-like pattern (b). At high power, branching tubules lined by clear epithelial cells (c) with adjacent spindle cell stroma (d). The epithelial component expresses NapsinA (e) and TTF1 (f)
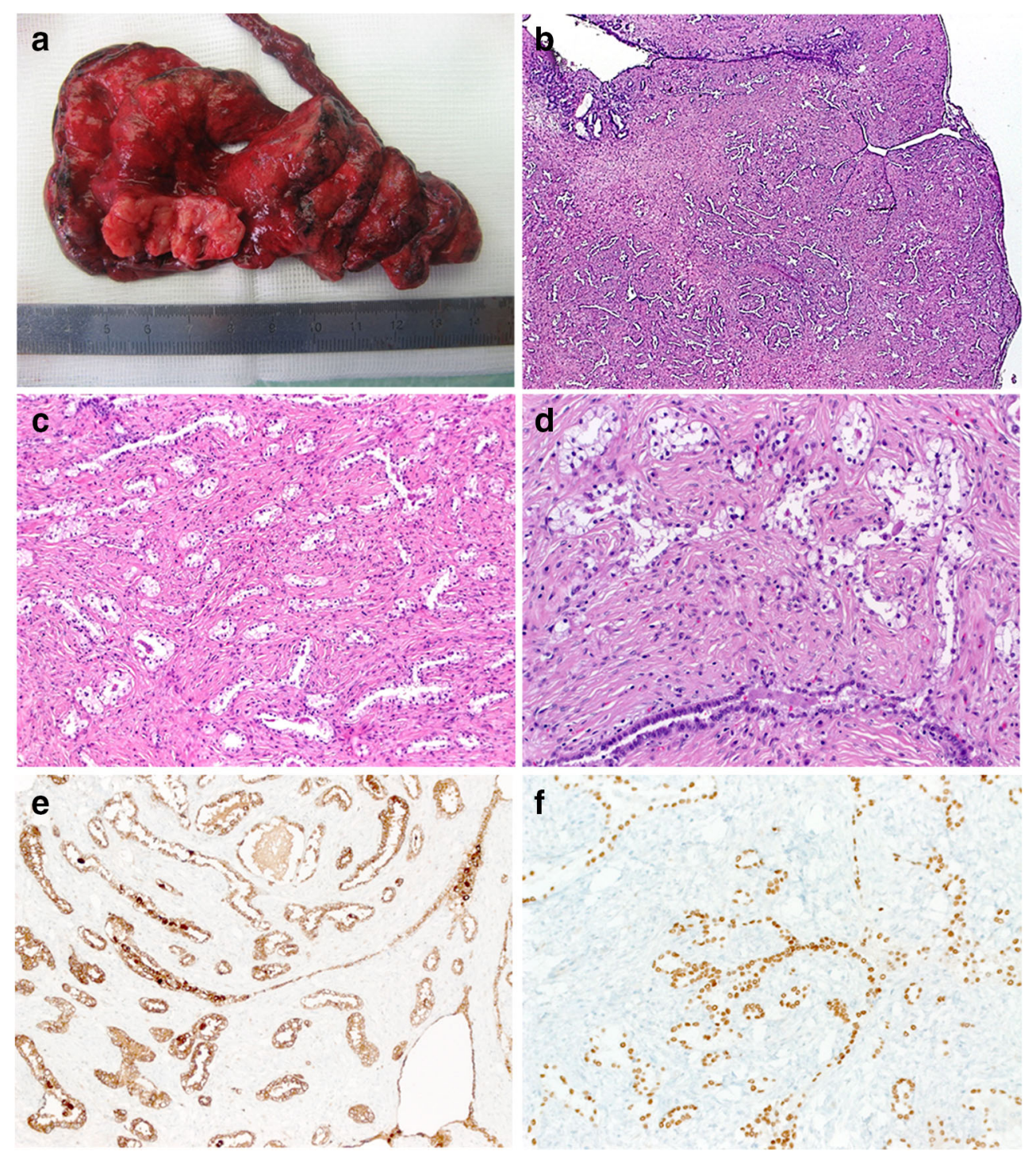

primary or metastatic), 4 metastases from different sarcoma types including multiple metastases from sclerosing epithelioid fibrosarcoma, 1 metastatic Wilms tumor, and 1 patient with two PAF-like lung nodules concurrent to a histologically identical mature testicular teratoma (Table 2). Notably, the Wilms tumor metastasis (post-treatment) contained prominent fibrous stroma entrapping an admixture of native respiratory epithelium as well as minute glands, positive for PAX8, negative with TTF1, indicating neoplastic epithelial origin. The metastatic sarcoma category contained different entities, but (except 1 leiomyosarcoma) all were of presumable fibroblastic histogenesis. Representative examples of these entities are illustrated in Fig. 2.

The two lung nodules from the patient with concurrent mature testicular teratoma showed concordant histology between the mesenchymal stromal component of both the testicular lesion and the lung nodules; both expressed desmin, smooth muscle actin (SMA), and pancytokeratin, but the epithelial components were discordant (Fig. $2 \mathrm{j}-1$ ). Although it is impossible to definitely classify the lung lesions of this patient without molecular testing for chromosome $12 p$ amplification, the concordant phenotypes and the clinical presentation are more consistent with metastatic disease but this remains unsolved.

Among the PAF-like lesions, only a single case in this group qualified as genuine PAF $(1 / 11 ; 9 \%)$. The spindled stroma of this case expressed SMA while the entrapped epithelium was positive for NapsinA and TTF1 (Fig. 1). Both of epithelial and stromal components were negative with estrogen and progesterone receptors, STAT6, and marker for adenocarcinoma of the lung (MAdL).

\section{Adenomyoepithelial-like pattern}

One case of metastatic atypical fibrous histiocytoma presented as a moderately cellular spindle cell lesion entrapping 

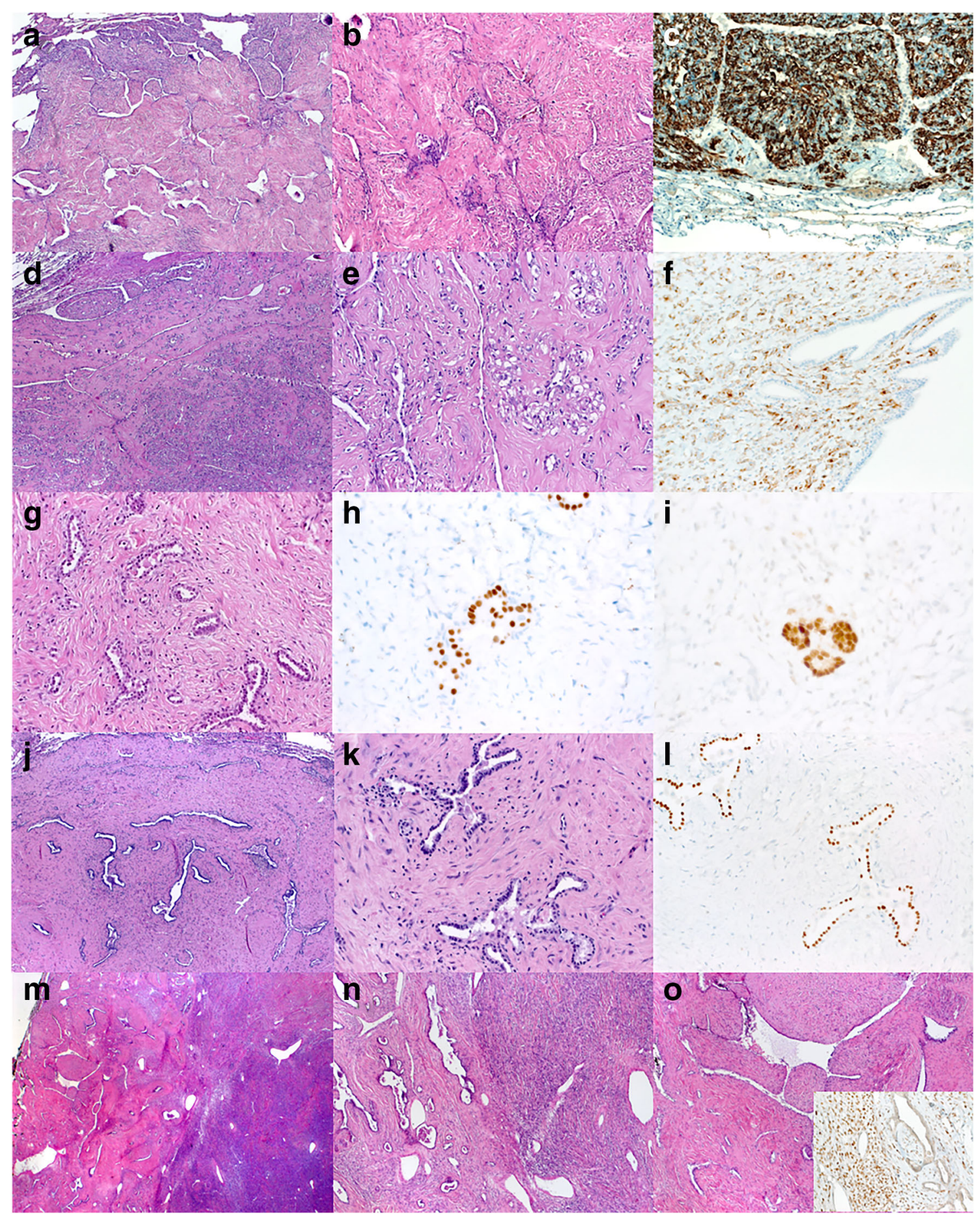

Fig. 2 Examples of primary and metastatic lung lesions closely mimicking (almost indistinguishable from) pulmonary adenofibroma. $\mathbf{a}-\mathbf{c}$ Lung metastasis of sclerosing epithelioid fibrosarcoma (a and $\mathbf{b}$ : H\&E; c: MUC4). d-f Intrapulmonary PEComa (d and e: H\&E; f: HMB45). g Metastatic Wilms tumor containing both TTF1+/PAX8entrapped respiratory epithelium (h) and PAX8+/TTF1- neoplastic

numerous small acinar respiratory glands mimicking adenomyoepithelioma (Fig. 3a). Diagnosis of metastatic atypical fibrous histiocytoma would have been impossible without knowing the clinical history. epithelium (i). $\mathbf{j}-\mathbf{l}$ Two adenofibroma-like lesions (j, $\mathbf{k}$ ) were detected at same time as a histologically identical mature testicular teratoma (j, $\mathbf{k}$ : H\&E; l: TTF1). Examples of cellular (m) and sclerosing (n) solitary fibrous tumor, both had prominent adenofibroma-like areas (seen on the left in both images). o Higher magnification of the adenofibroma-like area. Strong expression of STAT6 in the stromal cells is seen in $\mathbf{0}$ (inset)

\section{Biphasic synovial sarcoma-like pattern}

A highly cellular spindle cell sarcoma with prominent evenly distributed small glands mimicking biphasic synovial sarcoma 


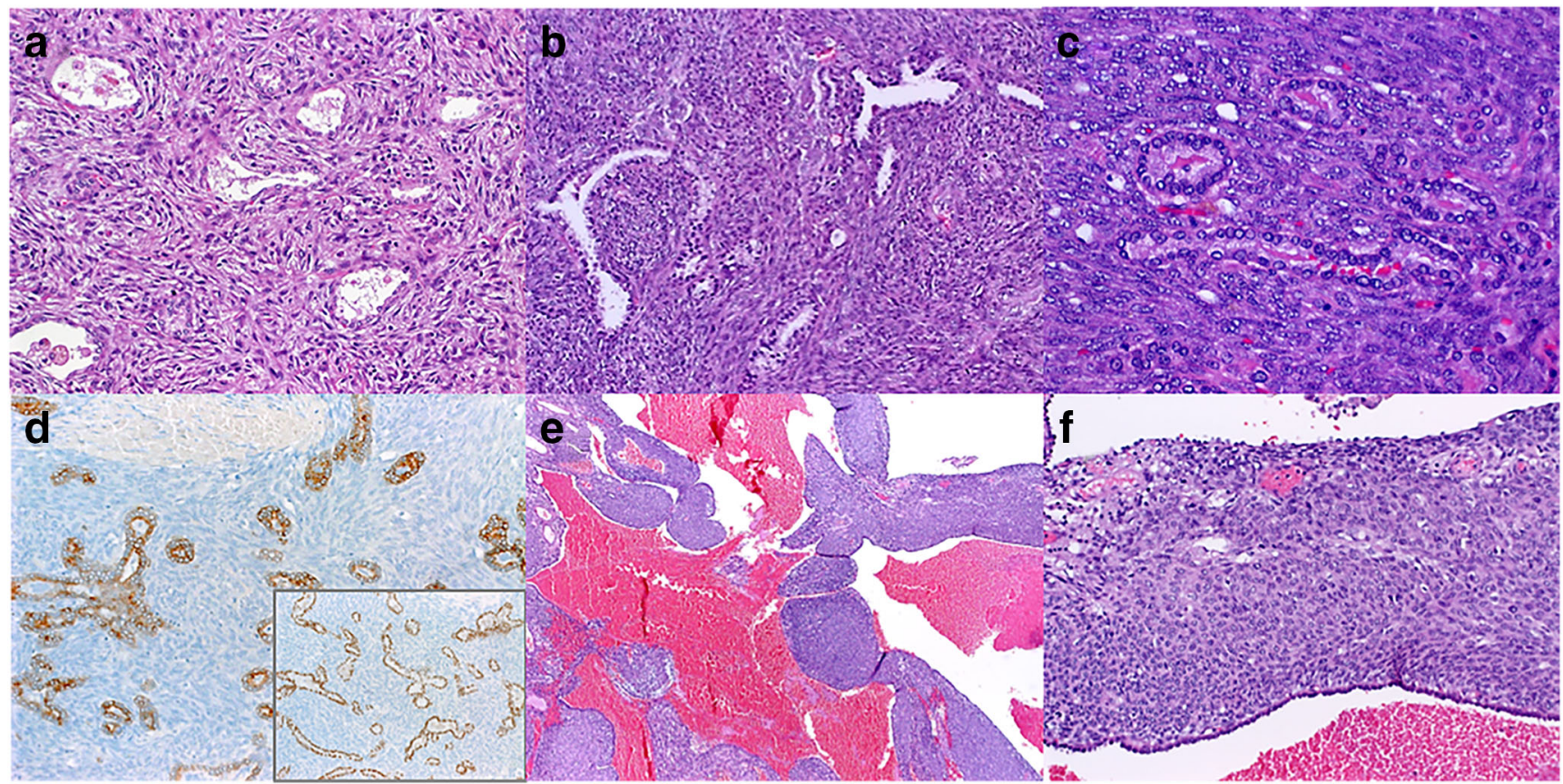

Fig. 3 Examples of metastatic mesenchymal neoplasms/sarcomas with prominent epithelial entrapment mimicking biphasic primary pulmonary neoplasms. Metastatic atypical fibrous histiocytoma with features mimicking adenomyoepithelioma (a). Metastatic unclassified spindle cell sarcoma (b) and monophasic spindle cell synovial sarcoma (c) mimicking biphasic synovial sarcoma. Expression of CK7 (d) and TTF1 (d inset) highlighting entrapped epithelium and confirming its pulmonary origin. e, f Lung metastasis of embryonal rhabdomyosarcoma displaying solid and cystic pattern closely mimicking type 2 pleuropulmonary blastoma was seen in two examples: one patient with multiple lung metastases of monophasic spindle cell synovial sarcoma (Fig. 3c) and another with lung metastasis from unclassified fibrosarcoma-like spindle cell sarcoma (Fig. 3b). Positive CK7 and TTF1 stains ruled out neoplastic epithelial elements in both cases (Fig. 3d).

\section{Pulmonary blastoma-like pattern}

This least common pattern was seen in a case of lung metastasis from embryonal rhabdomyosarcoma that contained prominent entrapped cystic spaces lined by respiratory epithelium closely mimicking type II pleuropulmonary blastoma (Fig. 3e, f). Notably, this case was subjected to NGS testing and lacked DICER1 mutations, thus arguing against the possibility of primary metachronous pleuropulmonary blastoma in the setting of DICER1 syndrome.

\section{Discussion}

It is well known that cancer metastasis may reveal diverse secondary morphological patterns that contribute to the differential diagnostic confusion in a given case. This is particularly true for lung metastasis where metastatic deposits may entrap native respiratory epithelium closely mimicking a biphasic neoplasm. The confusion is further enhanced by the frequent observation of prominent reactive and/ or regenerative changes of entrapped native epithelial glands closely mimicking neoplastic elements. Over the years, we have encountered several metastatic lung lesions that have been mistaken for primary pulmonary malignancies (carcinosarcoma, adenomyoepithelial carcinoma, etc.) based on prominent entrapped native glandular component where the alveolar epithelial immunophenotype was misinterpreted as evidence of pulmonary origin. This prompted us to perform the current study.

In the present study, we identified and illustrated different morphological patterns adopted by primary or metastatic nonepithelial neoplasms in the lung. Based on the degree of cellularity and other characteristics of the neoplastic mesenchymal component and the pattern of entrapped native respiratory epithelium, the tumors we have analyzed closely mimicked a variety of benign or malignant, primary or metastatic pulmonary neoplasms. Exploration of the previous clinical history/ imaging combined with careful assessment of the stromal characteristics for phenotypic hints was the key to correct diagnosis.

The biphasic pattern in lung metastasis, particularly from low-grade non-epithelial neoplasms/sarcomas, frequently obscures the original morphological pattern seen in the primary tumor and, instead, closely mimics benign or harmless hamartomatous lesions. Pulmonary adenofibroma (PAF) is the most frequent and the most striking and misleading pattern 


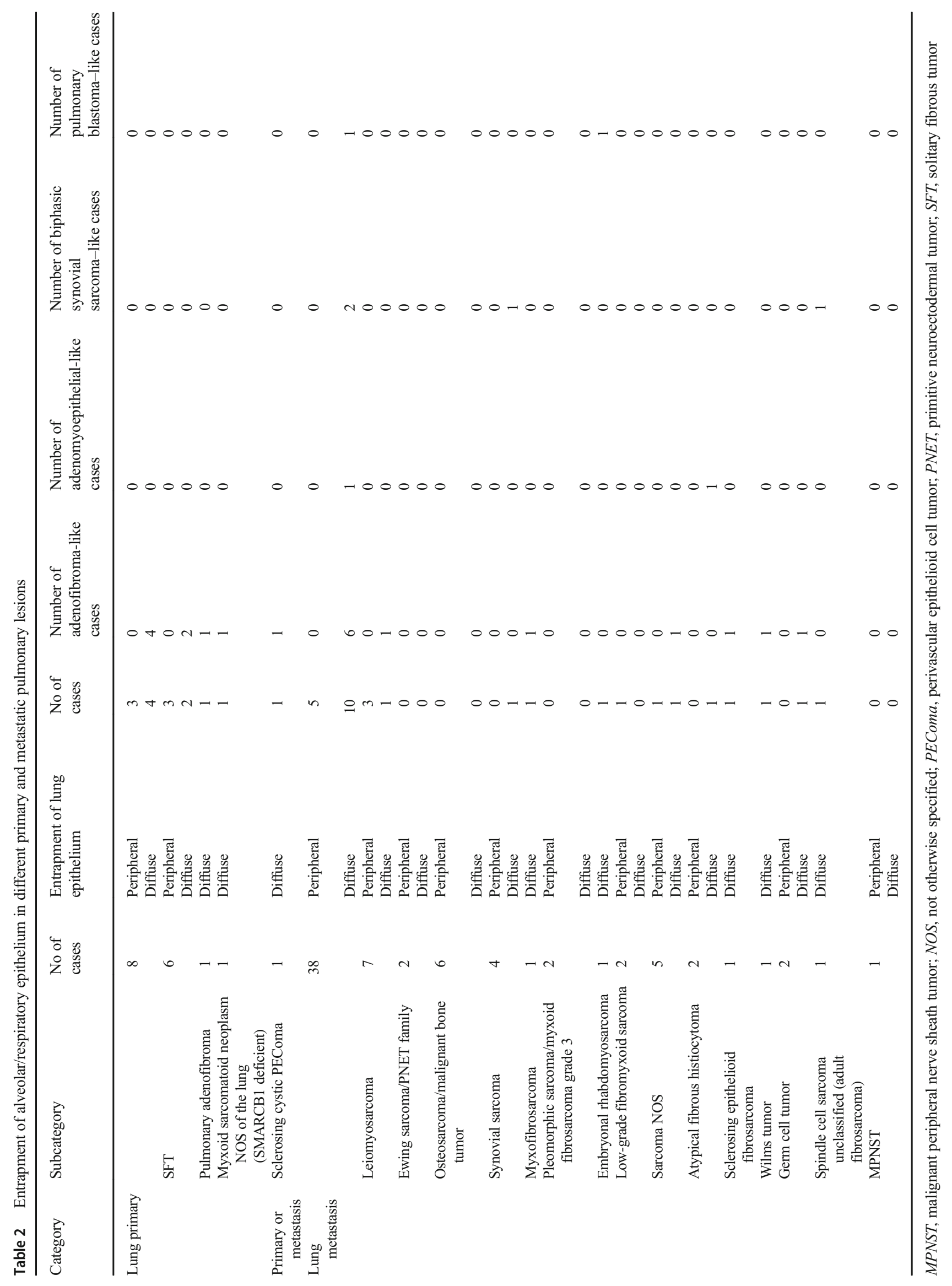


encountered in this study. Paucicellular intrapulmonary SFT is the main representative in this category. While some SFTs contain only focal PAF-like areas and are thus easily recognizable as adenofibromatous SFT variants, others were uniformly PAF-like. Their immunoprofile is otherwise indistinguishable from conventional SFTs. PAF-like SFTs represented up to $24 \%$ of intrapulmonary SFTs in a previous series [6]. Following discovery of STAT6-NAB2 gene fusions as driver events in most of SFTs, STAT6 IHC has emerged as highly sensitive and specific marker for SFT [7-9]. In the pre-STAT6 era however, many PAF-like SFTs were misclassified as PAF. This is because PAFs and SFTs otherwise share expression of CD99, CD34, bcl-2, and vimentin in their mesenchymal component $[5,10,11]$. Notably, $71 \%$ of PAFs were STAT6positive and showed NAB2-STAT6 rearrangement, confirming PAF-like SFT [5]. In a previous study, $71 \%$ of PAFs expressed hormone receptors in the stroma but all SFTs were negative for estrogen receptor- $\alpha$ by IHC [5]. Based on these recent observations and our current study, the concept of PAF as a specific entity is questionable as most of putative PAFs seem to be classifiable as other distinctive entities. The molecular pathogenesis and histogenesis of the vanishingly rare genuine "PAF lesions" remains to be further studied.

Other entities that closely mimicked PAF in this study include metastatic Wilms tumor, mature teratoma, and sclerosing epithelioid fibrosarcoma. Admittedly, many of these entities would have been impossible to diagnose by morphology alone if the clinical history was not available and/or STAT6 (in cases of intrapulmonary SFT) IHC was not applied.

In this context, it is worth mentioning that lung metastasis from biphasic neoplasms (Müllerian adenosarcomas and malignant fibroepithelial tumors of breast, prostate, etc.) usually contains only the mesenchymal stromal component. Biphasic synovial sarcoma may be the main exception to this. Accordingly, any glandular component in a biphasic lung lesion should be considered to represent entrapped native epithelium until proven otherwise. Immunophenotyping of the epithelial glandular component and careful assessment of atypia within the glands should allow distinguishing reactive entrapped glands from genuine neoplastic epithelial component.

On the other hand, SFTs with high cellularity and spindle cell sarcomas metastatic to the lung might be mistaken for biphasic synovial sarcoma. In contrast to biphasic synovial sarcoma, the entrapped alveolar glands show consistent pneumocytic phenotype, which is not the case in synovial sarcoma glands (the latter are CK7+, TLE1+, TTF1-, NapsinA-). Furthermore, the presence of alveolar-type glands with prominent regenerative atypia can closely mimic primary biphasic lung malignancies, in particular carcinosarcoma, primary and metastatic sarcomatoid (dedifferentiated) adenocarcinoma, and adenomyoepithelioma (epithelial-myoepithelial carcinoma). Carcinosarcoma (sarcomatoid carcinoma) typically features frankly malignant glands and highgrade cytology in both components, and the stromal component is usually highly pleomorphic. Pulmonary adenomyoepithelioma may represent primary lung neoplasm or metastasis from a salivary gland primary (epithelial-myoepithelial carcinoma) [12]. Recognition of the myoepithelial phenotype in the stromal component is helpful in diagnosis as well as the clinical history. In addition, the spatial arrangements of the epithelium surrounded by clear cell myoepithelial component are typical. In doubtful cases, it seems that HRAS mutation testing is context-specific for epithelial-myoepithelial carcinoma [12]. The rare pneumocytic adenomyoepithelioma, which contains similar alveolar-type neoplastic glands associated with myoepithelial stromal component, might be challenging. However, the neoplastic nature of the epithelial component in pneumocytic adenomyoepithelioma has been recently questioned [13].

In summary, we herein illustrated pitfalls related to frequent florid entrapment of native pulmonary epithelium within primary and metastatic non-epithelial neoplasms closely mimicking a biphasic lesion and occasionally leading to erroneous diagnosis of a primary pulmonary neoplasm based on the pneumocytic immunophenotype of the glandular component. As genuine PAF is exceptionally rare and a PAF-like pattern can be frequently seen in a variety of primary (benign or malignant) and metastatic lung tumors, any PAF-like lung lesion should be approached very critically and the recent and remote clinical history evaluated for any extrapulmonary neoplasm. Accordingly, PAF should be considered a diagnosis by exclusion. Misdiagnosis of metastatic malignancies as a "harmless PAF" would have significant prognostic and therapeutic implications.

Acknowledgments Special thanks to Gertrud Zimmermann and Natascha Leicht for technical support. Furthermore, we want to thank Dr. Denis Trufa for providing medical history of one case.

Authors' contribution AA, RE: conception and design of the work, acquisition, analysis and interpretation of data, drafting the MS and revising it critically for important intellectual content.

$\mathrm{FH}, \mathrm{AH}$ : interpretation of data, read and edited the MS critically for important intellectual content.

AA, RE, FH, AH: read and approved the final manuscript.

Funding Information Open Access funding provided by Projekt DEAL.

\section{Compliance with ethical standards}

Ethical responsibilities of authors section and compliance with ethical rules Samples were used in accordance with ethical guidelines for the use of retrospective tissue samples provided by the local ethics committee of the Friedrich-Alexander University Erlangen-Nuremberg (ethics committee statements 24.01.2005 and 18.01.2012). 
Conflict of interest The authors declare that they have no conflicts of interest.

Open Access This article is licensed under a Creative Commons Attribution 4.0 International License, which permits use, sharing, adaptation, distribution and reproduction in any medium or format, as long as you give appropriate credit to the original author(s) and the source, provide a link to the Creative Commons licence, and indicate if changes were made. The images or other third party material in this article are included in the article's Creative Commons licence, unless indicated otherwise in a credit line to the material. If material is not included in the article's Creative Commons licence and your intended use is not permitted by statutory regulation or exceeds the permitted use, you will need to obtain permission directly from the copyright holder. To view a copy of this licence, visit http://creativecommons.org/licenses/by/4.0/.

\section{References}

1. Suster S, Moran CA (1993) Pulmonary adenofibroma: report of two cases of an unusual type of hamartomatous lesion of the lung. Histopathology 23(6):547-551

2. Tan BY, Tan PH (2018) A diagnostic approach to fibroepithelial breast lesions. Surg Pathol Clin 11(1):17-42

3. Scarff RW, Gowar FJS (1944) Fibroadenoma of the lung. J Pathol Bacteriol 56:257-259

4. Kumar R, Desai S, Pai T, Pramesh CS, Jambhekar NA (2014) Pulmonary adenofibroma: clinicopathological study of 3 cases of a rare benign lung lesion and review of the literature. Ann Diagn Pathol 18(4):238-243

5. Fusco N, Guerini-Rocco E, Augello C, Terrasi A, Ercoli G, Fumagalli C, Vacirca D, Braidotti P, Parafioriti A, Jaconi M, Runza L, Ananthanarayanan V, Pagni F, Bosari S, Barberis M, Ferrero S (2017) Recurrent NAB2-STAT6 gene fusions and oestrogen receptor-alpha expression in pulmonary adenofibromas. Histopathology 70(6):906-917

6. Rao N, Colby TV, Falconieri G, Cohen H, Moran CA, Suster S (2013) Intrapulmonary solitary fibrous tumors: clinicopathologic and immunohistochemical study of 24 cases. Am J Surg Pathol 37(2):155-166

7. Doyle LA, Vivero M, Fletcher CD, Mertens F, Hornick JL (2014) Nuclear expression of STAT6 distinguishes solitary fibrous tumor from histologic mimics. Mod Pathol 27(3):390-395

8. Tan SY et al (2017) Solitary fibrous tumors in pediatric patients: a rare and potentially overdiagnosed neoplasm, confirmed by STAT6 immunohistochemistry. Pediatr Dev Pathol 21:1093526617745431

9. Barthelmess S et al (2014) Solitary fibrous tumors/ hemangiopericytomas with different variants of the NAB2STAT6 gene fusion are characterized by specific histomorphology and distinct clinicopathological features. Am J Pathol 184(4): 1209-1218

10. Sironi M, Rho B, Spinelli M (2005) Adenofibromatous pattern in a solitary fibrous tumor of the lung. Int J Surg Pathol 13(1):79

11. Cavazza A, Rossi G, de Marco L, Putrino I, Pellegrino S, Piana S (2003) Pseudopapillary solitary fibrous tumor of the lung: pulmonary fibroadenoma and adenofibroma revisited. Pathologica 95(3): 162-166

12. Hsieh MS, Chen JS, Lee YH, Chou YH (2016) Epithelialmyoepithelial carcinoma of the salivary gland harboring HRAS codon 61 mutations with lung metastasis. Int J Surg Pathol 24(3): 227-231

13. Yuan L, Katabi N, Antonescu CR, Golden A, Travis WD, Rekhtman N (2020) Pulmonary myoepithelial tumors with exuberant reactive pneumocytes: proposed reclassification of so-called pneumocytic adenomyoepithelioma. Am J Surg Pathol 44(1): $140-147$

Publisher's note Springer Nature remains neutral with regard to jurisdictional claims in published maps and institutional affiliations. 